\title{
Factors Associated with Opioid Use Disorder Diagnosis and Treatment Progress
}

\author{
Reanne Christina Mathai \\ University of Florida \\ Faculty mentor: Lisa J. Merlo, Department of Psychiatry
}

\begin{abstract}
Previous research has shown that various environmental aspects, such as a history of abuse, trauma, and pain can influence the incidence of opioid use disorder (OUD). The aims of this study were to determine risk factors associated specifically with opioid use disorder, and to identify factors that may predict treatment progress for opioid use disorder patients. Three-hundred six patients from an abstinence-based residential treatment center participated in this study. Participants completed a comprehensive battery, which included measures assessing quality of life, craving, abstinence self-efficacy, adverse childhood experiences, trauma exposure, and physical pain symptoms at treatment intake and again after 28 days of treatment. Independent t-test results showed significant differences between patients with and without opioid use disorders in social relationships, confidence abstaining from alcohol and drugs, pain intensity, and drug craving. Multiple regression analyses demonstrated that presence of an opioid use disorder predicted alcohol abstinence, social relationships, and baseline environment. Results revealed shifting attitudes towards substance use and social relationships during the opioid group's time in treatment. Cravings and urges were identified as important focuses for future treatment of opioid use disorder. Hyperalgesia was seen as an effect of extended opioid use and its impact decreased through drug abstinence. Limitations included a small sample size, a large proportion of wealthy individuals, and age differences between the two groups.

Keywords: Opioid use disorder, abstinence-based treatment, substance use disorder, predictors
\end{abstract}

\section{Background}

Although prescription opioids help to manage acute pain after medical procedures, they are highly addictive and subject to abuse. Misuse of prescription opioids can lead to development of an opioid use disorder, with many individuals eventually turning to use of heroin and/or illicit fentanyl as their opioid use disorder intensifies and their access to prescription opioids decreases. (National Academies of Sciences, Engineering, and Medicine, 2017). Serious consequences, including fatal overdose, are common. In recent years, there has been a significant increase in the number of opioid-related deaths. In 2016, over 42,000 people died of opioid overdose in the United States, up 28\% from 2015 (Hedegaard, Warner, \& Miniño, 2017). Moreover, these drugs impair daily functioning and can lead to blood-borne illnesses, including HIV infection and hepatitis (Skala et al., 2013). Patients with an opioid use disorder may put their career, family, 
and safety at risk to obtain and use the drugs (Cragg et al., 2017). With over eleven million people in the US having abused opioids in the past year (Volkow, 2017), this issue warrants increased attention.

Previous research involving individuals with substance use disorders (SUDs) has suggested that a number of biopsychosocial and socioeconomic factors may increase vulnerability to developing this condition (Boscarino et al., 2010; Brown et al., 2017; Cragg et al., 2017; Edlund, Steffick, Hudson, Harris, \& Sullivan, 2007; Wilsey et al., 2008). For example, research has demonstrated a link between the experience of physical pain and presence of an opioid use disorder (OUD). One study conducted with OUD patients found that they reported higher pain levels than participants in a control group (Ives et al., 2006). This is important because patients are often introduced to opioids for pain after a medical procedure (Ives et al., 2006) and extended use may result in development of both tolerance and physical dependence on the substance (Ives et al., 2006; Edlund et al., 2007; McCauley, Mercer, Brady, \& Back, 2014). The longer a patient has been using opioids, the more likely he or she is to develop a use disorder (Edlund et al., 2007). In another study, those who had been using opioids for 211 days or more had a statistically higher risk of developing OUD than those who had been using opioids for less time (Edlund et al., 2007). Patients who experience physical trauma and end up in the emergency room are twice as likely to develop opioid use disorder compared to members of the general population (Brown et al., 2017).

Psychological trauma, including physical, mental, emotional, and sexual abuse, has also been shown to increase the likelihood of developing a substance use disorder (Mirhashem, et al., 2017, Conroy, Degenhardt, Mattick, \& Nelson, 2009; Daigre et al., 2015; Dube et al., 2013; Heffernan et al., 2000), with just one additional traumatic event increasing a person's chances of developing a substance use disorder by $40 \%$ (Dube et al., 2013). Various studies have shown that the presence of emotional and physical abuse are the strongest predictors of developing a SUD, especially in men (Mirhashem, et al., 2017). Notably, while the experience of traumatic events in an individual's life can make him or her more susceptible to developing any SUD (Boscarino et al., 2010; McCauley et al., 2014), prescription opioid use, specifically, has been associated with higher scores on inventories measuring PTSD symptoms (Meier, Lambert-Harris, McGovern, Xie, \& McLeman, 2014). 
Further, though trauma can occur at any point during a patient's life, the most marked effects are shown when traumatic events occur during childhood (Conroy et al., 2009; Dube et al., 2013; Heffernan et al., 2000; Barahmand, Khazaee, \& Hashjin, 2016). In addition to any type of abuse a child might experience, parental separation, parental conflict, and parental substance abuse exponentially increase the rates of developing a SUD in the future (Conroy et al., 2009; Barahmand et al., 2016). Opioid use disorder has been specifically associated with childhood emotional abuse (Mirhashem, et al., 2017). Other forms of neglect and household dysfunction have a similar effect at these children age (Dube et al., 2013; Barahmand et al., 2016). Childhood mistreatment can increase the risk of substance use by 2-3 times (Heffernan et al., 2000).

One of the reasons trauma appears to have such a large impact on risk for SUD is due to the development of post-traumatic stress disorder (PTSD). It is common for patients with PTSD to also have diagnosis of general anxiety disorder and major depressive disorder, which are also positively correlated with the incidence of many substance use disorders (Skala et al., 2013; Boscarino et al., 2010; Mirhashem, et al., 2017; Daigre et al., 2015; Garami et al., 2017; Lai, Cleary, Sitharthan, \& Hunt, 2015; Wilsey et al., 2008; Robinson, Sareen, Cox, \& Bolton, 2009; Jacobsen, Southwick, \& Kosten, 2001; Dore, Mills, Murray, Teesson, \& Farrugia, 2012). Nonetheless, even when controlling for trauma and pain, some individuals appear to be at higher risk for developing a SUD than others. For example, previous research has shown that demographic risk factors include low socioeconomic status, low education level, being single, and being male (Skala et al., 2013; Edlund et al., 2007; Robinson et al., 2009; Dore et al., 2012). Some studies have shown that race may also have an impact on developing a SUD, but the results across studies have been inconclusive (Ives et al., 2006; Edlund et al., 2007; Robinson et al., 2009). In addition, multiple studies have found that one of the largest predictors of OUD specifically is past or present addiction to another substance (Skala et al., 2013; Boscarino et al., 2010; Ives et al., 2006; Lai et al., 2015; Domino et al., 2005). This seems to be due to the fact that many substance use disorders have similar risk factors, such as family history, mental illness, and trauma (Skala et al., 2013; Boscarino et al., 2010; Ives et al., 2006; Lai et al., 2015; Domino et al., 2005).

However, despite the extensive research done on risk factors for SUDs, and the drastically increasing prevalence of OUD, there has yet to be a study documenting psychosocial correlates exclusive to the development of OUD (alone or when co-occurring with other SUDs). Although 
studies of individuals with OUD exist, few studies have compared patients with OUD to those with other substance use disorders. Furthermore, no studies found have examined group differences among patients currently participating in abstinence-based residential treatment for SUD. The only studies found that address group differences are those that identify differences between substance use disorder patients and a control group.

Abstinence-based treatment requires patients to be admitted to inpatient programs while refraining from all illegal addictive substances. Most patients with OUD are treated in outpatient settings, using agonist (i.e., methadone) or partial agonist (buprenorphine) maintenance therapies (Rosenblum et al., 2003), resulting in a dearth of data regarding how patients with OUD fare in abstinence treatment compared to those with other SUDs. Indeed, in all previous studies found, the participants with OUD were recruited from methadone clinics. This introduces important limitations to the research because patients treated with methadone have been shown to report higher levels of pain compared to OUD patients in abstinence-based residential treatment facilities (Rosenblum et al., 2003). Additionally, methadone is a full $\mu$ opioid receptor agonist, meaning that the patients do not implement or maintain an opioid-free lifestyle during treatment (Rosenblum et al., 2003).

Therefore, the aims of this study were to identify: 1) psychosocial risk factors that are uniquely associated with seeking treatment for an opioid use disorder, and 2) predictors of treatment progress for individuals with opioid use disorders compared to patients with other substance use disorders. It was hypothesized that patients undergoing residential treatment for OUD would report higher pain intensity, increased history of emotional abuse, and higher rates of posttraumatic stress disorder compared to those with other SUDs. According to the literature, opioidinduced hyperalgesia can lead to an increased pain level after the prolonged use of opioid drugs (Silverman, Hansen, Lee, Patel \& Manchikanti, 2011). Therefore, OUD patients, who have been using these drugs for a long period of time may feel more pain due to this effect than non-OUD patients. Once OUD patients stop taking the opioids, their pain levels should subside (Silverman et al., 2011). Since methadone is an opioid, this would explain why patients treated in methadone clinics have higher levels of pain than OUD patients in abstinence-based programs. In addition to pain, secondary analyses examined other differences in predictors of treatment progress between the two groups as well.

\section{Methods}




\section{Measures}

The following measures were used in a comprehensive treatment battery within three days of patient admission to evaluate initial symptom severity. Each measure was completed again after 28 days of treatment, with the exception of the ACES and LEC-5, which assessed historical experiences that should not have changed over the previous 28 days. Differences between initial scores and follow-up scores were used to assess treatment progress.

demographics. Items assessed include age, gender, race/ethnicity, education level, occupation, and tobacco use history.

whoqol-bref. The WHOQOL-BREF is an inventory (World Health Organization (WHO), 1996) that measures the quality of a person's general, physical, psychological health, as well as their environment and social relationships over the past two weeks. General health measures patients' overall well-being. Physical health measures patients' ability to complete their daily activities. Psychological health measures patient life satisfaction. Environmental health measures the caliber of the patients' resources and living arrangements. Social relationships measures the quality of patients' personal relationships. There are 26 items in the WHOQOL-BREF inventory; each question corresponds to a specific subcategory. Each item is scored 1-5. A higher score indicates a higher quality of life. The WHOQOL-BREF was piloted and field tested around the world and has good psychometric properties.

penn alcohol craving scale (pacs). The PACS (Flannery, Volpicelli, \& Pettinati, 1999) is a 5item questionnaire that measures alcohol craving during the past week. This includes frequency, intensity, and duration of cravings as well as an indication of craving level overall. Each question is scored on a scale of 0-6. A higher score indicates a higher level of craving. The PACS has a high reliability, construct validity, discriminative validity, and predictive validity.

alcohol abstinence self-efficacy (aase). The AASE (DiClemente, Carbonari, Montgomery, \& Hughes, 1994) is a self-report measure that assesses patients' confidence in their ability to abstain from alcohol in various high risk situations. The four subscales include: Negative Affect, which measures abstinence when patients are feeling bad, Social/Positive, which measures confidence in abstaining in social situations, Physical and Other Concerns, which measures abstinence during physical distress, and Withdrawal and Urges, which measures ability to abstain despite cravings. Each subscale has 5 questions, for a total of 20 questions. Items are scored 
using a 1-5 Likert Scale, leading to scores ranging from 5-25 in each of the four categories. The higher the score, the more confident the patient is in their ability to abstain from alcohol.

drug abstinence self-efficacy (dase). The AASE was adapted for the present study to assess confidence ability to abstain from drug use.

post-traumatic stress disorder checklist (pcl-5). PCL-5 (Blevins, Weathers, Davis, Witte, \& Domino, 2015) was developed to assess DSM-5 symptoms of PTSD. It has displayed solid internal consistency $($ alpha $=0.94)$, high test-retest reliability $(\mathrm{r}=0.82)$, and strong convergent ( $r s=.74$ to 0.85 ) and discriminant validity (rs=.31 to 0.60 ). Each item is scored from $0-4$, with a total score ranging from 0-80. A higher score indicates a higher severity of PTSD symptoms.

adverse childhood experiences (ace) questionnaire. The ACE (Dube et al., 2001) is used to identify distressing experiences that individuals may have experienced during the first 18 years of their lives. Half of these include types of abuse: emotional, physical, verbal, sexual, and emotional neglect. The other 5 questions describe environmental conditions such as domestic violence, substance abuse by a household member, mental illness in household, parental separation or divorce, and parental incarceration. The ACE consists of 10 questions worth one point each. A higher score indicates more adverse experiences during childhood.

promis pain intensity. The PROMIS Pain Intensity (National Institutes of Health $(\mathrm{NIH})$, 2017) instrument was developed by the NIH to measure pain. The inventory consists of three items about the patient's pain during the past seven days. Each item is scored on a scale of 1-5, with a higher score indicating a higher pain intensity.

promis pain interference. The PROMIS Pain Interference (National Institutes of Health (NIH), 2018) instrument was developed by the NIH to measure how much an individual's pain disrupted other activities in their life over the past 7 days. The inventory consists of four items scored on a scale of 1-5, with a higher score indicating a higher level of interference.

life events checklist (lec-5). The LEC-5 (Weathers et al., 2013) is a 17-item inventory that assesses lifetime exposure to traumatic events. Patients are asked to indicate whether each event happened to them personally, they witnessed it happening to someone else, they learned about it, it happened as part of their job, they are not sure, or it does not apply to them. For this study, only events that happened to the individual personally were counted, creating a range of possible scores from 0-17, with a higher score indicating a higher number of traumatic events. 


\section{Procedure}

The experimental design was a case control study. Patients at a residential substance use disorder treatment facility completed the aforementioned measures as part of a comprehensive assessment administered within 3 days of intake as a part of their clinical standard of care. Instructions were given orally by a member of the assessment team, each of whom was trained to deliver the instructions using a script. The assessments were then reviewed by the assessment team member for missing data. Assessments were repeated when patients reached 28 days in treatment. Treatment consisted of a partial hospitalization program requiring an assigned individual therapist and group therapy. Patients were also able to attend optional supplementary activities, such as music therapy, cognitive behavioral therapy, and AA/NA meetings. Although optional, patients were highly encouraged to attend the supplementary activities, and did, so dosing of these and the required treatment were about the same in all participants. Those who wished to participate in the research study agreed to have their clinical assessment data exported anonymously for analysis. Consent to participate in the research study was obtained in a separate appointment, with $87 \%$ of patients providing consent. Treatment was not affected by their decision to participate in the research and no incentives were offered.

\section{Results}

Table 1. Patient Demographics: Highest Education Level

\begin{tabular}{lcc}
\hline Education Level & Number of Patients & Percentage \\
\hline Did not complete high school & 4 & 1.3 \\
GED & 12 & 3.9 \\
High school diploma & 30 & 9.8 \\
Some vocational/technical & 1 & 0.3 \\
training & 14 & 4.6 \\
Technical/vocational diploma & 64 & 20.9 \\
Some college & 35 & 11.4 \\
Associate degree & 63 & 20.6 \\
Bachelor's degree & 25 & 8.2 \\
Master's degree & 58 & 19.0 \\
Doctoral degree & & \\
\hline
\end{tabular}


Table 2. Patient Demographics: Occupation

\begin{tabular}{lcc}
\hline Job Sector & Number of Patients & Percentage \\
\hline Construction, Production, & 45 & 14.7 \\
Maintenance & & 6.9 \\
Management, Office and & 21 & 13.4 \\
Administrative & & \\
Sales, Business, Financial & 41 & 31.0 \\
Operations & & 2.9 \\
Healthcare & 95 & \\
Community, Social, & 9 & 4.2 \\
Protective Services & 13 & 2.9 \\
Education & 9 & 8.2 \\
Math, Science, Engineering & 25 & 2.3 \\
Food and Personal Care & 7 & 3.3 \\
Legal & 10 & 5.6 \\
Student & 17 & 1.6 \\
Unemployed & 5 & \\
Miscellaneous & & \\
\hline
\end{tabular}

Table 3. Patient Demographics: Substance Use Disorder Diagnosis

\begin{tabular}{lcc}
\hline Substance Use Disorder & Number of Patients & Percentage \\
\hline Alcohol & 157 & 51.3 \\
Opioid & 69 & 22.5 \\
Cannabis & 49 & 16.0 \\
Sedative/Hypnotic/Anxiolytic & 50 & 16.3 \\
Cocaine & 8 & 2.6 \\
Other Stimulant & 46 & 15.0
\end{tabular}

Note. Total exceeds $100 \%$ because some patients were diagnosed with more than one substance use disorder.

The sample $(\mathrm{N}=306)$ included 188 men $(61.4 \%), 117$ women $(38.2 \%)$, and one transgender individual $(0.3 \%)$. The mean age was 39.46 years (range $=18$ to 73 years) old. Two hundred eighty-eight $(94.1 \%)$ participants were Caucasian, eleven $(3.6 \%)$ were Black or African American, seven (2.3\%) were Asian, 19 (6.2\%) answered "other," with 26 (8.5\%) identifying as Hispanic or Latino. A total of 253 (83.0\%) patients had used tobacco in their lifetimes and 185 $(60.9 \%)$ were current users. Table 1 summarizes the education level of the patients in the sample. Table 2 describes their occupation distribution. Table 3 identifies the substance use disorders diagnoses of the patients in the sample.

The sample was divided into: 1) individuals with any opioid use disorder, whether or not they had a co-occurring substance use disorder involving alcohol or other drugs (OUD group), and 2) 
individuals with a substance use disorder that did not meet diagnostic criteria for OUD (nonOUD group), based on clinical interview by a physician specializing in addiction medicine. Independent samples t-tests comparing the non-OUD group and the OUD group demonstrated a significant age difference between groups. The mean ages for the opioid and non-opioid groups were 36.4 years and 40.4 years, respectively $(p=.04)$. There were no gender differences between groups, with 39 (20.7\%) of the 188 men and 30 (25.6\%) of the 117 women diagnosed with OUD. There were no significant differences between the groups in terms of education, race, ethnicity, or history of or current tobacco use.

Independent samples t-tests demonstrated significant differences between the OUD and nonOUD groups at baseline and after 28 days of treatment, with patterns suggesting different levels of initial symptom severity and treatment response. Initial symptom severity was indicated with the measures provided above. Treatment response was analyzed by comparing initial scores on the various measures to the scores at the 28-day follow-up. In terms of pain intensity, at baseline, the OUD group had significantly higher pain levels compared to the non-OUD group $(t=-2.114$, $p=.04)$. However, after 28 days, there were no significant differences between the opioid and non-opioid groups. Conversely, when examining craving, patients in both groups had similar scores at baseline; however, after 28 days of treatment, the non-OUD group showed a trend toward greater improvement, with the OUD group reporting slightly higher levels of cravings by comparison $(t=-1.902, p=.06)$. There were no significant differences between groups on selfreported exposure to adverse childhood experiences (ACE questionnaire), lifetime exposure to traumatic events (LEC-5), or symptoms of posttraumatic stress disorder (PCL-5).

With regard to abstinence self-efficacy, at baseline, the OUD group scored lower than the nonOUD group on the DASE Negative Affect subscale $(M=11.8$ vs. $M=14.6, t=2.404, p<.02)$, Physical Concerns subscale $(M=13.1$ vs. $M=16.5, t=3.160, p<.01)$, and Withdrawal and Urges subscale $(M=12.4$ vs. $M=15.7, t=3.015, p<.01)$. After 28 days, there were no differences between groups on the DASE Negative Affect subscale. However, the OUD group still reported significantly less abstinence self-efficacy related to DASE Withdrawal and Urges $(M=19.3$ vs. $M=21.2, t=1.981, p=.05)$ and showed a trend toward less abstinence selfefficacy related to DASE Physical Concerns $(t=1.884, p=.06)$. For the DASE Social/Positive subscale, the groups did not differ at baseline. However, after 28 days, the non-OUD group demonstrated a trend toward higher abstinence self-efficacy in this area $(t=1.941, p=.06)$. 
However, the OUD group reported higher alcohol abstinence self-efficacy than the non-OUD group ( $M=18.6$ vs. $M=15.3)$ on the AASE Withdrawal and Urges subscale $(t=-2.915, p<.01)$ and the AASE Negative Affect subscale ( $M=17.6$ vs. $M=14.1, t=-3.013, p<.01)$. The OUD group also demonstrated a trend toward higher alcohol abstinence self-efficacy on the AASE Physical Concerns subscale $(t=-1.861, p=.06)$. However, there were no significant differences between the groups on the AASE Social/Positivity subscale. Additionally, after 28 days in treatment, there were no differences between groups in alcohol abstinence self-efficacy for any subscale.

With regard to quality of life, there was no significant difference between the groups at baseline. However, at the 28 day follow-up, the OUD group $(M=15.3)$ had a significantly higher social relationships score than the non-OUD group $(M=14, t=-2.034, p=.04)$.

To compare treatment progress between groups, multiple regression analyses were used to predict scores following 28 days of treatment while controlling for baseline scores in step 1 and entering group designation (i.e., OUD group vs. non-OUD group) in step 2. Results demonstrated that improvement on the AASE Social/Positive subscale was significantly associated with group designation $\left(R^{2}=0.16, \Delta R^{2}=.03, p=.05\right)$. Additionally, improvement in scores on the WHOQOL-BREF environmental subscale were also significantly predicted by SUD group $\left(R^{2}=0.26, \Delta R^{2}=.02, p<.05\right)$. No other measures demonstrated significant results.

\section{Discussion}

Results of the current study provide interesting information regarding how the lived experience of SUD treatment may differ between individuals with OUD and those with other SUDs. There were no differences between groups in exposure to ACEs, trauma, or PTSD symptoms. This is mostly likely due to the fact that trauma has a similar effect on the development of all SUDs, not just OUD. Because past studies focused on comparing patients with SUDs to a control group, the differences between different SUDs could not be seen. Therefore, in this study, when comparing OUD and SUDs, it is able to be seen that the development of all SUDs are similarly affected by traumatic experiences.

On the other hand, consistent with previous research, the OUD group reported higher pain levels at baseline. However, this difference disappeared over 28 days of treatment, which may indicate abstinence-induced resolution of the hyperalgesia associated with prolonged usage of 
opioid drugs. As stated in previous research, this pain is often introduced during a medical procedure or trauma and perpetuated through opioid-induced analgesia. As a result, the initial hypothesis is only partially supported, as only pain severity was uniquely associated with OUD.

When used to estimate patient scores at follow-up, three areas were successfully predicted based on group designation: alcohol abstinence, social relationships, and the quality of their home environment. These results support the aforementioned literature. However, there were many other differences in treatment outcomes and treatment progress for OUD and non-OUD patients that proved to be significantly different for the two groups. Previous research has shown that patients with opioid use disorder tend to have more intense cravings and withdrawal symptoms. In this study, the OUD group reported lower drug abstinence self-efficacy at baseline, as well as higher craving and lower drug abstinence self-efficacy after 28 days of treatment. However, the OUD group did not display a generalized lack of self-efficacy. Indeed, they reported higher efficacy related to abstaining from alcohol at baseline, though this difference disappeared after 28 days of treatment as the OUD self-efficacy scores declined and resembled the scores of non-OUD patients. One explanation may be that these patients likely entered treatment to address their opioid usage, and may not have considered their alcohol use to be a major issue. However, during treatment, they learn about the importance of abstaining from all illicit mood-altering substances, putting them in a mindset similar to patients entering treatment for a primary alcohol use disorder or other SUD. With this frame of mind, OUD patients are more likely to take steps to reduce their alcohol usage as well, one of which is looking at the reality of their ability to abstain from it.

Interestingly, the OUD group reported greater improvements in their social relationships following 28 days of treatment. However, they also noted less improvement in their ability to abstain from drug use in social/celebratory situations. Often times, OUD patients have friends or family members who also use opioid drugs (Cragg, 2017; Domino, 2005). With these findings, it can be inferred that as the patients' relationships with their families and friends are improving, they may feel more pressure to engage in taking these drugs in a social setting. The reason this is more likely to happen for OUD patients than non-OUD patients is unknown, and could be an area of future research. 


\section{Conclusion}

As a result of the worsening opioid epidemic, the goals of this study were to identify risk factors unique to OUD and predictors of treatment progress for these patients compared to other SUD patients. It was hypothesized that pain severity, a history of emotional abuse, and the presence of PTSD symptoms would all be higher in OUD patients. However, with the use of a comprehensive psychometric assessment battery, it was determined that pain intensity and pain interference were the only risk factors unique to OUD from the original hypothesis, which was most likely introduced through some kind of trauma and continued through opioid-induced hyperalgesia. When analyses were done to assess treatment progress, they showed that OUD patients were less likely to take their alcohol usage seriously before they enter treatment, but began to as they progressed through treatment. They also tended to begin treatment at a lower self-efficacy of abstaining from their drugs, but increased to be on par with other non-OUD patients by the 28-day follow-up. These results show that abstinence-based treatment can be an effective option for OUD patients. However, OUD patients tend to struggle more with withdrawal and craving management. They also are less likely to believe they will abstain from drug use in social situations. Finally, multiple regression analyses found that that presence of an OUD at baseline predicted alcohol abstinence, social relationships, and baseline environment scores at follow-up. Finding a way to emphasize and effectively address these issues for OUD patients will be imperative to improving their overall treatment outcomes in the future.

\section{Limitations}

Although this study was able to add information to the body of knowledge surrounding different types of SUDs, there were still some limitations with it. The generalizability of the study may be limited by the fact that the sample was more highly-educated and less diverse than the general population, and all participants were able to afford at least 28 days of residential treatment for SUD. The small but significant age difference between the OUD and non-OUD may be a confounding factor influencing results. In addition, participants were recruited from a community treatment center, so groups were not entirely distinct. Many of those included in the OUD group also had other SUD diagnoses, and some participants may have been receiving medications to assist with relapse prevention (e.g., buprenorphine, naltrexone, acamprosate). The use of exclusively self-report measures also introduced potential biases, and limiting the data 
collection to only the first 28 days of treatment precludes the ability to make conclusions regarding treatment outcome. Finally, the relatively small sample, coupled with the large number of analyses conducted, increased the likelihood of Type I error.

In order to overcome these limitations, studies in the future should include a greater number of patients and look at the entirety of patients' time in treatment. Furthermore, analysis of a sample that represents individuals of lower socioeconomic status, especially those without insurance, may provide more robust, generalizable results.

\section{Acknowledgements}

This study was made possible through the University of Florida University Scholars Program. A special thanks to Dr. Lisa Merlo, Deborah Morrison, and the rest of the assessment team at the Florida Recovery Center for all the support and assistance.

\section{References}

Barahmand, U., Khazaee, A., \& Hashjin G.S. (2016). Emotion dysregulation mediates between childhood emotional abuse and motives for substance use. Archives of Psychiatric Nursing, 30(6), 653-659. doi: https://doi.org/10.1016/j.apnu.2016.02.007

Blevins, C.A., Weathers, F.W., Davis, M.T., Witte, T.K., \& Domino, J.L. (2015). The posttraumatic stress disorder checklist for DSM-5 (PCL-5): Development and initial psychometric evaluation. Journal of Traumatic Stress, 28(6), 489-498. doi: https://doi.org/10.1002/jts.22059

Boscarino, J.A., Rukstalis, M., Hoffman, S.N., Han, J.J., Erlich, P.M., Gerhard, G.S., \& Stewart, W.F. (2010). Risk factors for drug dependence among out-patients on opioid therapy in a large US health-care system. Addiction, 105(10), 1776-1782. doi: 10.1111/j.1360-0443.2010.03052.x.

Brown, R., Deyo, B., Riley, C., Quanbeck, A., Glass, J.E., Turpin, R., ... Agarwal, S. (2017). Screening in trauma for opioid misuse prevention (STOMP): study protocol for the development of an opioid risk screening tool for victims of injury. Addiction Science \& Clinical Practice, 12(1), 2836. doi: 10.1186/s13722-017-0097-6.

Conroy, E., Degenhardt, L., Mattick, R.P., \& Nelson, E.C. (2009). Child maltreatment as a risk factor for opioid dependence: comparison of family characteristics and type and severity of child maltreatment with a matched control group. Child Abuse and Neglect, 33(6), 343-352. doi: https://doi.org/10.1016/j.chiabu.2008.09.009

Cragg, A., Hau, J.P., Woo, S.A., Liu, C., Doyle-Waters, M.M., \& Hohl, C.M. (2017). Risk factors for addiction among patients receiving prescribed opioids: a systematic review protocol. Systematic Reviews, 6(1), 265-272. doi: 10.1186/s13643-017-0642-0

Daigre, C., Rodríguez-Cintas, L., Tarifa, N., Rodríguez-Martos, L., Grau-López, L., Berenguer, M. ..., Roncero, C. (2015). History of sexual, emotional or physical abuse and psychiatric comorbidity 
in substance-dependent patients. Psychiatry Research, 229(3), 743-749. doi:

https://doi.org/10.1016/j.psychres.2015.08.008

DiClemente, C.C., Carbonari, J.P., Montgomery, R.P.G. \& Hughes, S.O. (1994).The Alcohol Abstinence Self-Efficacy Scale. Journal of Studies on Alcohol, 55(2), 141-148.

Domino, K.B., Hornbein, T.F., Polissar, N.L.,Renner, G., Johnson, J., Alberti, S., \& Hankes, L. (2005). Risk factors for relapse in health care professionals with substance use disorders. Journal of the American Medical Association, 293(12), 1453-1460. doi: 10.1001/jama.293.12.1453

Dore, G., Mills, K., Murray, R., Teesson, M., \& Farrugia, P. (2012). Post-traumatic stress disorder, depression and suicidality in inpatients with substance use disorders. Drug and Alcohol Review, 31(3), 294-302. doi: https://doi.org/10.1111/j.1465-3362.2011.00314.x

Dube, S.R., Felitti, V.J., Dong, M., Chapman, D.P., Giles, W.H., \& Anda, R.F. (2013). Childhood abuse, neglect, and household dysfunction and the risk of illicit drug use: The adverse childhood experiences study. Pediatrics, 111(3), 564-572. doi: 10.1542/peds.2015-4016

Edlund, M.J., Steffick, D., Hudson, T., Harris, K.M., \& Sullivan, M. (2007). Risk factors for clinically recognized opioid abuse and dependence among veterans using opioids for chronic non-cancer pain. Pain, 129(3), 355-362. doi: 10.1016/j.pain.2007.02.014

Flannery, B.A., Volpicelli, J.R., \& Pettinati, H.M. (1999). Psychometric properties of the Penn Alcohol Craving Scale. Alcoholism, Clinical and Experimental Research, 23(8), 1289-1295.

Hedegaard, H., Warner, M., \& Miniño, A.M. (2017, December 21). Drug overdose deaths in the United States, 1996-2016. Retrieved from https://www.cdc.gov/nchs/data/databriefs/db294.pdf

Heffernan, K., Cloitre, M., Tardiff, K., Marzuk, P.M., Portera, L., \& Leon, A.C. (2000). Childhood trauma as a correlate of lifetime opiate use in psychiatric patients. Addictive Behaviors, 25(5), 797-803. doi: https://doi.org/10.1016/S0306-4603(00)00066-6

Ives, T. J., Chelminski, P. R., Hammett-Stabler, C. A., Malone, R. M., Perhac, J. S., Potisek, N. M., ... Pignone, M. P. (2006). Predictors of opioid misuse in patients with chronic pain: a prospective cohort study. BMC Health Services Research, 6(1), 46. doi: 10.1186/1472-6963-6-46

Jacobsen, L.K., Southwick, S.M.,\& Kosten, T.R., (2001). Substance use disorders in patients with posttraumatic stress disorder: A review of the literature. The American Journal of Psychiatry, 158(8), 1184-1190. doi: 10.1176/appi.ajp.158.8.1184

Lai, H.M.X., Cleary, M., Sitharthan, T \& Hunt, G.E. (2015). Prevalence of comorbid substance use, anxiety and mood disorders in epidemiological surveys, 1990-2014: A systematic review and meta-analysis. Drug Alcohol Dependence, 154(1), 1-13. doi: 10.1016/j.drugalcdep.2015.05.031.

McCauley, J., Mercer, M.A., Brady, K.T., \& Back, S.E. (2014). Trauma histories of non-treatmentseeking prescription opioid-dependent individuals. Drug and Alcohol Dependence, 140(1), e139e140. doi: https://doi.org/10.1016/j.drugalcdep.2014.02.396

Meier, A., Lambert-Harris, C., McGovern, M.P., Xie, H., \& McLeman, B. (2014). Co-occurring prescription opioid use problems and posttraumatic stress disorder symptom severity. The American Journal of Drug and Alcohol Abuse, 40(4), 304-311. doi: 10.3109/00952990.2014.910519. 
Mirhashem, R., Allen, H.C., Adams, Z.W., van-Stolk-Cooke, K., Legrand, A., \& Price, M. (2017). The intervening role of urgency on the association between childhood maltreatment, PTSD, and substance-related problems. Addictive Behaviors, 69(1), 98-103. doi:

https://doi.org/10.1016/j.addbeh.2017.02.012

National Academies of Sciences, Engineering, and Medicine. (2017). Pain management and the opioid epidemic: Balancing societal and individual benefits and risks of prescription opioid use. Washington, DC: National Academies Press.

National Institutes of Health. (2017). PROMIS Pain Intensity Scoring Manual [PDF file]. Retrieved from http://www.healthmeasures.net/images/PROMIS/manuals/PROMIS_Pain_Intensity_Scoring_Ma nual.pdf

National Institutes of Health. (2018). PROMIS Pain Interference Scoring Manual [PDF file]. Retrieved from http://www.healthmeasures.net/images/PROMIS/manuals/PROMIS_Pain_Interference_Scoring Manual.pdf

Rampakakis, E., Ste-Marie, P. A., Sampalis, J. S., Karellis, A., Shir, Y., \& Fitzcharles, M. A. (2015). Real-life assessment of the validity of patient global impression of change in fibromyalgia. $R M D$ Open, 1(e000146). doi:10.1136/rmdopen-2015-000146.

Robinson, J., Sareen, J., Cox, B., \& Bolton, J. (2009). Self- medication of anxiety disorders with alcohol and drugs: Results from a nationally representative sample. Journal of Anxiety Disorders, 23(1), 38-45. doi: https://doi.org/10.1016/j.janxdis.2008.03.013

Rosenblum, A., Joseph, H., Fong, C., Kipnis, S., Cleland, C.,\& Portenoy, R.K. (2003). Prevalence and characteristics of chronic pain among chemically dependent patients in methadone maintenance and residential treatment facilities. Journal of the American Medical Association, 289(18), 23702378. doi: 10.1001/jama.289.18.2370

Skala, K., Reichl, L., Ilias, W., Likar, R., Grögl-Aringer, G., Wallner, C...Walter, H. (2013). Can we predict addiction to opioid analgesics? A possible tool to estimate the risk of opioid addiction in patients with pain. Pain Physician, 16(1), 593-601.

Silverman, S. M., Hansen, H., Lee, M., Patel, V. B., \& Manchikanti, L. (2011). A comprehensive review of opioid-induced hyperalgesia. Pain Physician, 14(2), 145-161.

Volkow, N. (2017). Federal efforts to combat the opioid crisis: a status update on CARA and other initiatives [Transcript]. Retrieved from https://www.drugabuse.gov/about-nida/legislativeactivities/testimony-to-congress/2017/federal-efforts-to-combat-opioid-crisis-status-update-caraother-initiatives

Weathers, F.W., Blake, D.D., Schnurr, P.P., Kaloupek, D.G., Marx, B.P., \& Keane, T.M. (2013). The Life Events Checklist for DSM-5 (LEC-5). Instrument available from the National Center for PTSD at www.ptsd.va.gov

Wilsey, B.L., Fishman, S.M., Tsodikov, A., Ogden, C., Symreng, I.,\& Ernst, A (2008). Psychological comorbidities predicting prescription opioid abuse among patients in chronic pain presenting to the emergency department. Pain Medicine, 9(8), 1107-17. doi: https://doiorg.lp.hscl.ufl.edu/10.1111/j.1526-4637.2007.00401.x

World Health Organization. (1996). WHOQOL-BREF: Introduction, administration, scoring and generic version of the assessment. Geneva, Switzerland: WHOQOL group. 
